\title{
sGC activators and stimulators attenuate ischemia/reperfusion injury of the lung
}

\author{
Bakytbek Egemnazarov*1, Akylbek Sydykov1, Ralph T Schermuly ${ }^{1,2}$, \\ Norbert Weissmann ${ }^{1}$, Johannes-Peter Stasch ${ }^{3}$, Werner Seeger ${ }^{1}$, \\ Friedrich Grimminger ${ }^{4}$ and Hossain A Ghofrani ${ }^{1}$
}

\author{
Address: ${ }^{1}$ Department of Internal Medicine II, University Hospital Giessen, Giessen, Germany, ${ }^{2}$ Max-Planck-Institute for Heart and Lung Research, \\ Bad Nauheim, Germany, ${ }^{3}$ Pharma Research Center, Bayer Healthcare, Wuppertal, Germany and ${ }^{4}$ Department of Internal Medicine II and V, \\ University Hospital Giessen, D-35392 Giessen, Germany \\ Email: Bakytbek Egemnazarov* - bakytbek.egemnazarov@innere.med.uni-giessen.de \\ * Corresponding author
}

from 4th International Conference of cGMP Generators, Effectors and Therapeutic Implications

Regensburg, Germany. 19-21 June 2009

Published: II August 2009

BMC Pharmacology 2009, 9(Suppl I):PI3 doi:I0.II86/|47|-22I0-9-SI-PI3

This abstract is available from: http://www.biomedcentral.com/I47I-22 I0/9/SI/PI3

(c) 2009 Egemnazarov et al; licensee BioMed Central Ltd.

\section{Background}

Protective effects of $\mathrm{NO}$, a physiological activator of soluble guanylyl cyclase (sGC), have been reported in ischemia/reperfusion syndrome $(\mathrm{I} / \mathrm{R})$ of the lung. However, its protective effects can be overwhelmed by its deleterious effects due to oxidative products formation. Therefore, we studied the effects of direct sGC stimulator (BAY 41-2272) and sGC activator (BAY 58-2667) on I/R injury of the lung in a isolated intact organ model.

\section{Materials and methods}

Lung injury was assessed by measurements of weight gain and microvascular permeability (capillary filtration coefficient (Kfc)). Production of reactive oxygen species (ROS) in tissue and release into the perfusate were measured during early reperfusion by ESR spectroscopy. BAY 41-2272, BAY 58-2667, or apocynin were applied 5 min before ischemia according to the protocol. NO was admixed into the inspiration loop of the ventilator at a dose of $20 \mathrm{ppm}$ 1 min before reperfusion.

\section{Results}

In untreated lungs dramatic rise in Kfc values and weight gain during reperfusion were observed. This was associated with increased ROS production. NO, BAY 41-2272, and BAY 58-2667 significantly attenuated vascular leak- age and suppressed ROS release. In an additional set of experiments BAY 41-2272 diminished PMA induced ROS production by NADPH-oxidases. Involvement of ROS generated by NADPH-oxidases in I/R was demonstrated by favorable effects of enzyme inhibition by apocynin. Moreover, NADPH oxidase activity, as measured in the membrane fractions from lung homogenates, was reduced in the BAY 41-2272 treatment group. NO protected against vascular leakage, however with less prominent effects as compared to sGC activator and stimulator.

\section{Conclusion}

Thus, sGC stimulator and activator protect against $\mathrm{I} / \mathrm{R}$ induced lung injury. Partly, this effect can be explained by prevention of NADPH oxidase activation. sGC stimulators and activators demonstrated better protection against $\mathrm{I} / \mathrm{R}$ induced lung injury compared to inhalative NO. 